\title{
Asymptotic Behavior of Higher Order Quasilinear Neutral Difference Equations
}

\author{
V. Sadhasivam ${ }^{1}$, Pon. Sundar ${ }^{2} \&$ A. Santhi ${ }^{1}$ \\ ${ }^{1}$ PG and Research Department of Mathematics, Thiruvalluvar Government Arts College, Rasipuram, Namakkal - 637 \\ 401, Tamil Nadu, India \\ 2 Om Muruga College of Arts and Science, Salem - 636 303, Tamil Nadu, India
}

Correspondence: V. Sadhasivam, PG and Research Department of Mathematics, Thiruvalluvar Government Arts College, Rasipuram, Namakkal - 637 401, Tamil Nadu, India. E-mail: ovsadha@gmail.com

Received: October 12, 2016 Accepted: November 21, 2016 Online Published: November 29, 2016

doi:10.5539/jmr.v8n6p148 URL: http://dx.doi.org/10.5539/jmr.v8n6p148

\begin{abstract}
We study, the asymptotic behavior of solutions to a class of higher order quasilinear neutral difference equations under the assumptions that allow applications to even and odd-order difference equations with delayed and advanced arguments, as well as to functional difference equations with more complex arguments that may for instance, alternate infinitely between delayed and advanced types. New theorems extend a number of results reported in the literature. Illustrative examples are presented.
\end{abstract}

Keywords: asymptotic behavior, higher order, quasilinear, even and odd order, delay and advanced arguments, functional difference equation

\section{Introduction}

In this paper, we study the asymptotic behavior of solutions to a class of higher-order quasilinear neutral functional difference equations

$$
\Delta\left(r(n)\left(\Delta^{m-1} z(n)\right)^{\alpha}\right)+q(n) x^{\beta}(\sigma(n))=0
$$

where $n \geq n_{0} \in N_{0}=\left\{n_{0}, n_{0}+1, n_{0}+2, \cdots\right\}, z(n)=x(n)+p(n) x(\tau(n))$ and $\Delta$ is the forward difference operator defined by

$$
\Delta x(n)=x(n+1)-x(n) \text { and } \Delta^{i} x(n)=\Delta\left(\Delta^{i-1} x(n)\right), \quad i=1,2, \cdots, m-1 .
$$

We assume the following conditions on equation (1), without further mention

$\left(c_{1}\right)\{r(n)\},\{\sigma(n)\}$ and $\{\tau(n)\}$ are positive sequences of real numbers such that $\Delta r(n) \geq 0, r(n)>0, \lim _{n \rightarrow \infty} \sigma(n)=\infty$.

$\left(c_{2}\right)\{p(n)\}$ and $\{q(n)\}$ are sequences of positive real numbers such that $q(n) \geq 0$ and $q(n)$ does not vanish eventually.

$\left(c_{3}\right) \alpha$ and $\beta \in \mathfrak{R}$ where $\mathfrak{R}$ stands for the set consisting of all quotients of odd positive integers.

Analysis of qualitative properties of equation (1) is important not only for the sake of further development of the oscillation theory, but for practical reasons too. In fact, an Emden-Fowler type differential equation

$$
\left(r(t)\left(x^{(n-1)}(t)\right)^{\alpha}\right)^{\prime}+q(t) x^{\beta}(\sigma(t))=0
$$

has numerous applications in physics and engineering: that is; for instance, the papers by Ou and Wong [2004].

By a solution of equation (1), we mean a real sequence $\{x(n)\}$ which is defined for $n \geq-\mu=\max \{\sup \{\tau(n), \sigma(n)\}$ and which satisfies the equation (1) for $n \geq n_{0}$. We deal only with proper solution $x(n)$ of (1) that satisfy the condition $\sup \{|x(n)|: n \geq N\}>0$ for all $N \geq N_{\mu}$ and tacitly assume that (1) possesses such solutions. A solution of (1) is said to be oscillatory if it has arbitrarily large zeros on $n \geq N_{\mu}$. Otherwise, it is termed nonoscillatory.

For several decades, an increasing interest in obtaining sufficient conditions for oscillatory and nonoscillatory behavior of different classes of difference equations has been observed; see, for instance, the monographs [Agarwal, 1992; Agarwal and Wong, 1997; Elaydi, 1995; Gyori and Ladas, 1991; Lakshmikanthan and Trigiante, 1988], the papers [Graef et al, 1996; Guan and Yang, 1999; He, 1993; Jurang and Bin, 1997; Karpuz, 2009; Sundar and Kishorkumar, 2014; Wong, 
1975; Yu and Wang, 1994; Zafer, 1995] and the references cited therein. Let us briefly comment on a number of related results which motivated our study.

S.S. Cheng and W.T. Patula [1993] studied the difference equation

$$
\Delta\left(\Delta y_{k-1}\right)^{p-1}+s_{k} y_{k}^{p-1}=0
$$

where $p>1$ and proved an existence theorem for the equation (3).

X.Zhou and J. Yan [1998] studied the difference equation

$$
\Delta\left[p_{n-1}\left(\Delta y_{n-1}\right)^{\delta}\right]+s_{n} y_{n}^{\delta}=0
$$

and they obtained some comparison results and necessary and sufficient conditions for the oscillation of equation (4).

I. Kvbiaczyk and S.H. Sekar [2002] studied the second order sublinear delay difference equation

$$
\Delta\left(p_{n}\left(\Delta x_{n}\right)\right)+q_{n} x_{n-\tau}^{\gamma}=0, \quad 0 \leq \gamma \leq 1 .
$$

By using Riccati transformation techniques the authors obtained oscillation criteria for the equation (5) under the conditions

$$
\sum_{n=n_{0}}^{\infty} \frac{1}{p_{n}}=\infty \quad \text { and } \quad \sum_{n=n_{0}}^{\infty} \frac{1}{p_{n}}<\infty
$$

Y. Bolat and O. Alzabot [2012] considered the half-linear delay difference equation

$$
\Delta\left[p_{n}\left(\Delta^{m-1}\left(x_{n}+q_{n} x_{\tau(n)}\right)\right)^{\alpha}\right]+\gamma_{n} x_{\sigma_{n}}^{\beta}=0, \quad n \geq n_{0}
$$

under the condition $\sum_{n_{0}}^{\infty} \frac{1}{\left(p_{n}\right)^{1 / \alpha}}<\infty$ and with using that $\Delta p_{n} \geq 0$ and derived some oscillation and asymptotic criteria for the equation (6).

M.K. Yildiz and O. Ocalan [2007] studied the neutral difference equation

$$
\Delta^{m}\left(y_{n}-p_{n} y_{n-k}\right)+q_{n} y_{n-l}^{\alpha}=0, \quad n \geq n_{1}
$$

where $1>\alpha>0$ is a quotient of odd positive integers and $\left\{p_{n}\right\}$ satisfies $-1<p_{n}<1$.

J. Luo in 2002 [2002] considered the second order quasilinear neutral delay difference equation

$$
\Delta\left[a_{n-1}\left|\Delta\left(x_{n-1}+p_{n-1} x_{n-1-\sigma}\right)\right|^{\alpha-1} \Delta\left(x_{n-1}+p_{n-1} x_{n-1-\sigma}\right)\right]+q_{n} f\left(x_{n-\tau}\right)=0
$$

under the condition $\sum^{\infty} \frac{1}{a_{n}^{1 / \alpha}}=\infty$ and obtained several oscillation results.

Pon. Sundar and E. Thandapani [2000], considered the second order quasilinear functional difference equation

$$
\Delta\left(|\Delta y(n)|^{\alpha-1} \Delta y(n)\right)+f(n, y(\sigma(n)))=0, \quad n \geq n_{0}
$$

and established some necessary and sufficient conditions for the equation (9) to have various types of nonoscillatory solutions.

Our principal goal is to analyze the asymptotic behavior of solutions to (1) in the case where the condition

$$
\sum_{n_{0}}^{\infty} \frac{1}{(r(n))^{1 / \alpha}}<\infty
$$

holds. We provide sufficient conditions which ensure that solution to (1) are either oscillatory or approach zero at infinity. In some cases, we reveal oscillatory nature of (1).

As usual, all functional inequalities are supposed to hold for all $t$ large enough. Without loss of generality, we deal only with positive solutions of (1) since, under our assumptions, if $x(n)$ is a solution, then $-x(n)$ is a solution of this equation too. 
In the sequel, we denote by $\tau^{-1}$ the function which is inverse to $\tau$. We also adopt the following notations for the compact presentation of our results.

$$
\begin{aligned}
A(n) & =\sum_{n}^{\infty} \frac{1}{(r(n))^{1 / \alpha}} \\
Q(n) & =\min \{q(n), q(\tau(n))\} \\
R(n) & =\max \{r(n), r(\tau(n))\} \\
Q_{\gamma}(n) & =Q(n)\left(\frac{\left(\eta_{1}(n)\right)^{m-1}}{\gamma^{1 / \alpha}\left(\eta_{1}(n)\right)}\right)^{\gamma} \\
Q_{\beta}(n) & =Q(n)\left((\sigma(n))^{(m-2)}\right)^{\beta}, \quad \tilde{Q}_{\beta}(n)=Q(n)\left(\frac{\left(\eta_{1}(n)\right)^{(m-1)}}{\gamma^{1 / \beta}\left(\eta_{1}(n)\right)}\right)^{\beta} \\
Q_{\theta}(n) & =Q(n)\left(\sum_{\eta_{3}(n)}^{\infty}\left(\eta-\eta_{3}(n)\right)^{(m-3)} A(\eta(n))\right)^{\theta} \\
\bar{Q}_{\beta}(n) & =Q(n)\left(\sum_{\eta_{3}(n)}^{\infty}\left(\eta-\eta_{3}(n)\right)^{(m-3)} A(\eta(n))\right)^{\beta} \\
\widehat{Q}_{\beta}(n) & =Q(n)\left(\frac{\sigma_{n}^{(m-1)}}{\gamma^{1 / \beta}(\sigma(n))}\right)^{\beta}, \quad \tilde{Q}_{\gamma}(n)=Q(n)\left(\frac{\sigma^{(m-1)}(n)}{\gamma^{1 / \alpha}(\sigma(n))}\right)^{\gamma}
\end{aligned}
$$

where the meaning of $\gamma, \theta, \eta_{i}$ and $\eta_{3}$ will be explained later.

\section{Asymptotic Behavior of Solutions to Even-order Equations}

In what follows, $\tau(n)$ can be both a delayed or an advanced argument. Throughout this section, in addition to the basic assumptions listed in the introduction, it is also supposed that (10) holds along with

$\left(c_{4}\right) 0 \leq p(n) \leq p_{0}<\infty$ for some constant $p_{0}$;

(c $\left.c_{5}\right) \Delta \tau(n) \geq \tau_{*}>0$ and $\tau \circ \sigma=\sigma \circ \tau$

We shall need the following lemmas which are useful is the sequel.

Lemma 1. [Gyori and Ladas, 1991] Assume that $q(n) \geq 0$ for all $n \in N$ and

$$
\lim \inf _{n \rightarrow \infty}\left[\sum_{n-l}^{n-1} q(s)\right]>\left(\frac{l}{l+1}\right)^{l+1} .
$$

Then,

(i) $v(n+1)-v(n)+q(n) v(n-l) \leq 0, n \in N$ has no eventually positive solution.

(ii) $v(n+1)-v(n)+q(n) v(n-l) \geq 0, n \in N$ has no eventually negative solution

(iii) $v(n+1)-v(n)+q(n) v(n-l)=0$ is oscillatory.

Lemma 2. [Agarwal, 1992](Discrete Kingser's Theorem) Let $z(n)$ be defined for $n \geq a$, and $z(n)>0$ with $\Delta^{m} z(n)$ of constant sign for $n \geq a$ and not identically zero. Then, there exists an integer $j, 0 \leq j \leq m$ with $(m+j)$ odd for $\Delta^{m} z(n) \leq 0$ and $(m+j)$ even for $\Delta^{m} z(n) \geq 0$, such that

$$
j \leq m-1 \text { implies }(-1)^{j+i} \Delta^{i} z(n)>0, \text { for all } n \geq a, j \leq i \leq m-1,
$$

and

$$
j \geq 1 \text { implies } \Delta^{i} z(n)>0 \text { for all large } n \geq a, 1 \leq i \leq j-1 \text {. }
$$


Lemma 3. [Agarwal, 1992] Let $z(n)$ be defined for $n \geq a$ and $z(n)>0$ with $\Delta^{m} z(n) \leq 0$ for $n \geq a$ and not identically zero. Then, there exists a large $n_{1} \geq a$ such that

$$
z(n) \geq \frac{\left(n-n_{2}\right)^{(m-1)}}{(m-1) !} \Delta^{(m-1)} z\left(2^{m-j-1}\right)(n), \quad n \geq n_{1},
$$

where $\lambda=\left(\frac{1}{2^{m-1}}\right)^{(m-1)}$ and $j$ is defined us in Lemma 2. Further $z(n)$ is increasing, then

$$
z(n) \geq \frac{\lambda}{(m-1) !} n^{(m-1)} \Delta^{m-1} z(n), \quad n \geq 2^{m-1} n .
$$

Lemma 4. Assume that $A \geq 0, B \geq 0, \alpha \geq 1$. Then

$$
(A+B)^{\alpha} \geq A^{\alpha}+B^{\alpha} .
$$

Proof. If $A=0$ or $B=0$, then the inequality holds trivially. For $A \neq 0$, setting $x=\frac{B}{A}$. The inequality takes the form $(1+x)^{\alpha} \geq 1+x^{\alpha}$ which is for $x>0$ evidently true.

Lemma 5. Assume that $A \geq 0, B \geq 0,0<\alpha \leq 1$. Then

$$
(A+B)^{\alpha} \geq \frac{A^{\alpha}+B^{\alpha}}{2^{1-\alpha}} .
$$

Proof. We may assume that $0<A<B$. Consider the function $f(n)=u^{\alpha}$. Since $\Delta^{\alpha} f(n)<0$ for $n>0$, the sequence $f(n)$ is concave down; that is

$$
f\left(\frac{A+B}{2}\right) \geq \frac{f(A)+f(B)}{2}
$$

which implies the desired inequality.

Theorem 6. Let $m \geq 2$ be even and $0<\beta \leq 1$. Assume that conditions $\left(c_{4}\right)$ and $\left(c_{5}\right)$ are satisfied, and there exist two real numbers $\gamma, \lambda \in R$ such that $\gamma \leq \beta \leq \lambda$ and $\gamma<\alpha<\lambda$. Suppose further that there exist two sequence $\eta_{1}^{(n)}, \eta_{2}^{(n)}$ such that

$$
\begin{gathered}
\eta_{1}(n) \leq \sigma(n) \leq \eta_{2}(n), \quad \eta_{1}(n)<n<\tau(n) \leq \eta_{2}(n) \\
\lim _{n \rightarrow \infty} \eta_{1}(n)=\infty .
\end{gathered}
$$

If

$$
\sum^{\infty} Q_{\gamma}(n)=\infty
$$

and

$$
\sum^{\infty} Q_{\beta}(n) \Delta^{\lambda}\left(\eta_{2}(n)\right)=\infty
$$

Then every solution $x(n)$ of (1) is either oscillatory or satisfies

$$
\lim _{n \rightarrow \infty} x(n)=0 .
$$

Proof. Assume that equation (1) has a nonoscillatory solutions $x(n)$ which is eventually positive and such that

$$
\lim _{n \rightarrow \infty} x(n) \neq 0 .
$$

Then $z(n)$ satisfies

$$
\begin{aligned}
z(\sigma(n)) & =x(\sigma(n))+p(\sigma(n)) x(\tau(\sigma(n))) \\
& \leq x(\sigma(n))+p_{0} x(\tau(\sigma(n))) .
\end{aligned}
$$


In view of (1), we have

$$
0=p_{0}^{\beta} \Delta\left[r(\tau(n))\left(\Delta^{m-1} z(\tau(n))\right)^{\alpha}\right]+p_{0}^{\beta} q(\tau(n)) x^{\beta}(\sigma(\tau(n)))
$$

Using (22), we obtain

$$
\begin{aligned}
& q(n) x^{\beta}(\sigma(n))+p_{0}^{\beta} q(\tau(n)) x^{\beta}(\sigma(\tau(n))) \\
& =q(n) x^{\beta}(\sigma(n))+p_{0}^{\beta} q(\tau(n)) x^{\beta}(\tau(\sigma(n))) \\
& \quad \geq Q(n) z^{\beta}(\sigma(n)) .
\end{aligned}
$$

It follows from equation (1), (22) and (23) that

$$
\Delta\left[r(n)\left(\Delta^{m-1} z(n)\right)^{\alpha}+p_{0}^{\beta} r(\tau(n))\left(\Delta^{m-1} z(\tau(n))\right)^{\alpha}\right]+Q(n) z^{\beta}(\sigma(n)) \leq 0 .
$$

Then there exist two possible cases:

(i)

$$
z(n)>0, \Delta^{m-1} z(n)>0, \Delta^{m} z(n) \leq 0, \Delta\left[r(n)\left(\Delta^{m-1} z(n)\right)^{\alpha}\right] \leq 0
$$

(ii)

$$
z(n)>0, \Delta^{m-2} z(n)>0, \Delta^{m-1} z(n)<0, \Delta\left[r(n)\left(\Delta^{m-1} z(n)\right)^{\alpha}\right] \leq 0
$$

for $n \geq n_{1}$ where $n_{1} \geq n_{0}$ is large enough.

Case 1: Suppose first that conditions (25) hold. Using inequality (24) and assumption $\eta_{1}(n) \leq \sigma(n)$, we conclude that

$$
\Delta\left[r\left(n\left(\Delta^{m-1} z(n)\right)^{\alpha}\right)+p_{0}^{\beta} r(\tau(n))\left(\Delta^{m-1} z(\tau(n))\right)^{\alpha}\right]+Q(n) z^{\beta}\left(\eta_{1}(n)\right) \leq 0 .
$$

Furthermore, by the monotonicity of $z(n)$, there exists a constants $M>0$ such that

$$
z^{\beta}\left(\eta_{1}(n)\right)=z^{\beta-\gamma}\left(\eta_{1}(n)\right) z^{\gamma}\left(\eta_{1}(n)\right) \geq M^{\beta-\gamma} z^{\gamma}\left(\eta_{1}(n)\right) .
$$

Combining (27) and (28), we have

$$
\Delta\left[r(n)\left(\Delta^{m-1} z(n)\right)^{\alpha}+p_{0}^{\beta} r(\tau(n))\left(\Delta^{m-1} z(\tau(n))\right)^{\alpha}\right]+M_{1} Q(n) z^{\gamma}\left(\eta_{1}(n)\right) \leq 0,
$$

where $M_{1}=M^{\beta-\gamma}$. An application of conditions (25) allows us to deduce that the sequence

$$
w(n)=r(n)\left(\Delta^{m-1} z(n)\right)^{\alpha}
$$

is positive and nonincreasing. By Lemma 3, we have

$$
\begin{aligned}
z(n) & \geq \frac{\lambda n^{(m-1)}}{(m-1) ! r^{1 / \alpha}(n)} r^{1 / \alpha} \Delta^{m-1} z(n) \\
& =\frac{\lambda n^{(m-1)}}{(m-1) ! r^{1 / \alpha}} w^{1 / \alpha}(n)
\end{aligned}
$$

for every $\lambda \in(0,1)$ and for sufficiently large $n$. Using (31) in (29), we conclude that $w(n)$ is a positive solution of a delay difference inequality

$$
\Delta\left(w(n)+p_{0} w(\tau(n))\right)+M_{1}\left(\frac{\lambda}{(m-1) !}\right)^{\gamma} Q_{r}(n) w^{\gamma / \alpha}\left(\eta_{1}(n)\right) \leq 0 .
$$

Define now a function $y(n)$ by

$$
y(n)=w(n)+p_{0} w(\tau(n)) .
$$


Then by the monotonicity of $w(n)$,

$$
y(n) \leq w(n)\left(1+p_{0}^{\beta}\right) .
$$

Substituting (34) into (32), we observe that $y(n)$ is a positive solution of a delay difference inequality

$$
\Delta y(n)+M_{1}\left(\frac{\lambda}{(m-1) !}\right)^{\gamma}\left(\frac{1}{1+p_{0}^{\beta}}\right)^{\gamma / \alpha} Q_{\gamma}(n) y^{\gamma / \alpha}\left(\eta_{1}(n)\right) \leq 0 .
$$

Then, by virtue of [Gyori and Ladas, 1991], the associated delay difference equation

$$
\Delta y(n)+M_{1}\left(\frac{\lambda}{(m-1) !}\right)\left(\frac{1}{1+p_{0}^{\beta}}\right) Q_{\gamma}(n) y^{\gamma / \alpha}\left(\eta_{1}(n)\right)=0
$$

also has a positive solution. However, by Lemma 1 implies that, under assumption (17), equation (36) is oscillatory. Therefore, (1) cannot have positive solutions.

Case 2: Assume now that conditions (26) hold. By virtue of (20), we have that

$$
\lim _{n \rightarrow \infty} z(n) \neq 0 .
$$

An application of Lemma 3 yields

$$
z(n) \geq \frac{\lambda}{(m-2) !} n^{(m-2)} \Delta^{m-2} z(n)
$$

for any $\lambda \in(0,1)$ and for sufficiently large $n$. Hence, by (24) and (38), we obtain

$$
\Delta\left[r(n)\left(\Delta^{m-1} z(n)\right)^{\alpha}+p_{0}^{\beta} r(\tau(n))\left(\Delta^{m-1} z(\tau(n))\right)^{\alpha}\right]+\left(\frac{\lambda}{(m-2) !}\right)^{\beta} Q_{\beta}(n)\left(\Delta^{m-2} z(\sigma(n))\right)^{\beta} \leq 0 .
$$

Using conditions $\Delta^{m-1} z(n)<0, \sigma(n) \leq \eta_{2}(n)$, and inequality (39), we have

$$
\Delta\left[r(n)\left(\Delta^{m-1} z(n)\right)^{\alpha}+p_{0}^{\beta} r(\tau(n))\left(\Delta^{m-1} z(\tau(n))\right)^{\alpha}\right]+\left(\frac{\lambda}{(m-2) !}\right)^{\beta} Q_{\beta}(n)\left(\Delta^{m-2} z\left(\eta_{1}(n)\right)\right)^{\beta} \leq 0 .
$$

Furthermore, by the monotonicity of $\Delta^{m-2} z(n)$, there exists a constant $N>0$ such that

$$
\begin{aligned}
\left(\Delta^{m-2} z\left(\eta_{1}(n)\right)\right)^{\beta} & =\left(\Delta^{m-2} z\left(\eta_{1}(n)\right)\right)^{\beta-\lambda}\left(\Delta^{m-2} z\left(\eta_{1}(n)\right)\right)^{\lambda} \\
& \geq N^{\beta-\lambda}\left(\Delta^{m-2} z\left(\eta_{1}(n)\right)\right)^{\lambda}
\end{aligned}
$$

Combining (40) and (41), we arrive at

$$
\Delta\left[r(n)\left(\Delta^{m-1} z(n)\right)^{\alpha}+p_{0}^{\beta} r(\tau(n))\left(\Delta^{m-1} z(\tau(n))\right)^{\alpha}\right]+N_{1}\left(\frac{\lambda}{(m-2) !}\right)^{\beta} Q_{\beta}(n)\left(\Delta^{m-2} z\left(\eta_{2}(n)\right)\right)^{\lambda} \leq 0
$$

where $N_{1}=N^{\beta-\lambda}$. Using the monotonicity of $w(n)$, for $s \geq n \geq n_{1}$ we conclude that

$$
r^{1 / \alpha}(s) \Delta^{m-1} z(s) \leq r^{1 / \alpha} \Delta^{m-1} z(n) .
$$

Dividing (43) by $r^{1 / \alpha}(s)$ and summing the resulting inequality from $n$ to $l-1$, we obtain

$$
\Delta^{m-2} z(l) \leq \Delta^{m-2} z(n)+r^{1 / \alpha}(n) \Delta^{m-1} z(n) \sum_{n}^{l-1} \frac{1}{r^{1 / \alpha}(s)} .
$$

Passing to the limit as $l \rightarrow \infty$, we deduce that

$$
0 \leq \Delta^{m-1} z(n)+r^{1 / \alpha} \Delta^{m-1} z(n) A(n)
$$


which yields

$$
\Delta^{m-2} z(n) \geq-A(n) r^{1 / \alpha}(n) \Delta^{m-1} z(n)=-A(n) w^{1 / \alpha}(n)
$$

Combining (42) and (46), we have

$$
\begin{aligned}
\Delta & {\left[r(n)\left(\Delta^{m-1} z(n)\right)^{\alpha}+p_{0}^{\beta} r(\tau(n))\left(\Delta^{m-1} z(\tau(n))\right)^{\alpha}\right] } \\
& -N_{1}\left(\frac{\lambda}{(m-2) !}\right)^{\beta} Q_{\beta}(n) A^{\lambda}\left(\eta_{2}(n)\right) w^{\lambda / \alpha}\left(\eta_{2}(n)\right) \leq 0
\end{aligned}
$$

Using the monotonicity of $w(n)$, we conclude that

$$
y(n) \geq w(\tau(n))\left[1+p_{0}^{\beta}\right]
$$

Using (48) into (47), we observe that $y(n)$ is a negative solution of an advanced difference inequality

$$
\Delta y(n)-N_{1}\left(\frac{\lambda}{(m-2) !}\right)^{\beta}\left(\frac{1}{1+p_{0}^{\beta}}\right)^{\lambda / \alpha} Q_{\beta}(n) A^{\lambda}\left(\eta_{2}(n)\right) A^{\lambda}\left(\eta_{2}(n)\right) y^{\lambda / \alpha}\left(\tau^{-1}\left(\eta_{2}(n)\right)\right) \leq 0
$$

which implies that $u(n)=-y(n)$ is a positive solution of an advanced difference inequality

$$
\Delta u(n)-N_{1}\left(\frac{\lambda}{(m-2) !}\right)^{\beta}\left(\frac{1}{1+p_{0}^{\beta}}\right)^{\lambda / \alpha} Q_{\beta}(n) A^{\lambda}\left(\eta_{2}(n)\right) A^{\lambda}\left(\eta_{2}(n)\right) u^{\lambda / \alpha}\left(\tau^{-1}\left(\eta_{2}(n)\right)\right)=0
$$

consequently, by virtue of [Gyori and Ladas, 1991] the associated advanced difference equation

$$
\Delta u(n)-N_{1}\left(\frac{\lambda}{(m-2) !}\right)^{\beta}\left(\frac{1}{1+p_{0}^{\beta}}\right)^{\lambda / \alpha} Q_{\beta}(n) A^{\lambda}\left(\eta_{2}(n)\right) A^{\lambda}\left(\eta_{2}(n)\right) u^{\lambda / \alpha}\left(\tau^{-1}\left(\eta_{2}(n)\right)\right)=0
$$

also has a positive solution. However, by [Sundar and Murugesan, 2010] implies that, under assumption (18) equation (51) is oscillatory. Therefore, (1) cannot have a positive solution this contradiction with initial assumption completes the proof.

Theorem 7. Let $m \geq 2$ be even, and let $0<\alpha=\beta \leq 1$. Assume that conditions ( $\left.c_{4}\right)$ and $\left(c_{5}\right)$ hold, and there exist two sequences $\eta_{1}(n), \eta_{2}(n)$ satisfying (16). Suppose also that conditions

$$
\frac{1}{((m-1) !)^{\beta}} \frac{1}{\left(1+p_{0}^{\beta}\right)} \lim _{n \rightarrow \infty} \sum_{\eta_{1}(n)}^{n-1} \tilde{Q}_{\beta}(n)>\left(\frac{k}{k+1}\right)^{k+1}
$$

where $k$ is the delay argument and

$$
\frac{1}{((m-2) !)^{\beta}} \frac{1}{\left(1+p_{0}^{\beta}\right)} \lim _{n \rightarrow \infty} \sum_{n}^{\tau^{-1}\left(\eta_{2}(n)\right)-1} Q_{\beta}(s) A^{\beta}\left(\eta_{2}(s)\right)>\left(\frac{l-1}{l}\right)^{l}
$$

where $l$ is the advance argument are satisfied. Then conclusion of Theorem 6 remains in fact.

Proof. Assume that $x(n)$ is an eventually positive solution of equation (1) that satisfies (20). Proceeding as in the proof of Theorem 6 , one comes to the conclusion that, for every $\lambda \in(0,1)$, a delay difference equation

$$
\Delta y(n)+\left(\frac{\lambda}{(m-1) !}\right)^{\beta} \frac{1}{1+p_{0}^{\beta}} \tilde{Q}_{\beta}(n) y\left(\eta_{1}(n)\right)=0
$$

and an advanced difference equation

$$
\Delta y(n)-\left(\frac{\lambda}{(m-2) !}\right)^{\beta} \frac{1}{1+p_{0}^{\beta}} Q_{\beta}(n) A^{\beta}\left(\eta_{2}(n)\right) u\left(\tau^{-1}\left(\eta_{1}(n)\right)\right)=0
$$

both have positive solutions. On the other hand, condition (52) and [Gyori and Ladas, 1991] imply that equation (54) is oscillatory, a contradiction. Likewise, by virtue of [Sundar and Murugesan, 2010, Lemma 2.3.2] condition (53) yields that equation (55) is oscillatory. This contradiction completes the proof. 
Theorem 8. Let $m \geq 2$ be even and $0<\beta \leq 1$. Assume that conditions $\left(c_{4}\right)$ and $\left(c_{5}\right)$ are satisfied, and there exist two numbers $\lambda, \gamma \in R$ as in Theorem 6 and two real sequences $\eta_{1}(n), \eta_{2}(n)$ such that

$$
\eta_{1}(n) \leq \sigma(n) \leq \eta_{2}(n), \quad \eta_{1}(n)<\tau(n) \leq n<\eta_{2}(n), \quad \lim _{n \rightarrow \infty} \eta_{1}(n)=\infty .
$$

If conditions (17) and (18) hold, the conclusion of Theorem 6 remains infact.

Proof. As above let $x(n)$ be an eventually positive solution of equation (1) that satisfies (20). As in the proof of Theorem 6 , we split the arguments into two parts.

Case 1: Assume first that (25) is satisfied. If has been established in the proof of Theorem 6 that the sequence $w(n)$ defined by (30) is positive, nonincreasing and satisfies inequality (32). Introducing again $y(n)$ by (33) and using the monotonicity of $w(n)$, we conclude that

$$
y(n) \leq w(\tau(n))\left(1+p_{0}^{\beta}\right)
$$

substituting of (57) into (32) implies that, for sufficiently large $n, y(n)$ is a positive solution of a delay difference inequality

$$
\Delta y(n)+M_{1}\left(\frac{\lambda}{(m-1) !}\right)^{\gamma}\left(\frac{1}{1+p_{0}^{\beta}}\right)^{\gamma / \alpha} Q_{\gamma}(n) y^{\gamma / \alpha}\left(\tau^{-1} \eta_{1}(n)\right) \leq 0
$$

Then, the associated difference equation

$$
\Delta y(n)+M_{1}\left(\frac{\lambda}{(m-1) !}\right)^{\gamma}\left(\frac{1}{1+p_{0}^{\beta}}\right)^{\gamma / \alpha} Q_{\gamma}(n) y^{\gamma / \alpha}\left(\tau^{-1} \eta_{1}(n)\right)=0
$$

also has a positive solution. However, by [Sundar and Murugesan, 2010, Lemma 2.3.2] implies that , under assumption (17) equation (59) is oscillatory. Therefore equation (1) cannot have positive solutions.

Case 2: Assume that (26) is satisfied. It has been established in the proof of Theorem 6 that the sequence $w(n)$ defined by (30) is negative, nonincreasing and satisfies the inequality (47). Introducing again $y(n)$ by (33) and using the monotonicity of $w(n)$, we conclude that

$$
y(n) \geq w(n)\left(1+p_{0}^{\beta}\right)
$$

substituting (60) into (47), we observe that $y(n)$ is a negative solution of an advanced difference inequality

$$
\Delta y(n)-N_{1}\left(\frac{\lambda}{(m-2) !}\right)^{\beta}\left(\frac{1}{1+p_{0}^{\beta}}\right)^{\lambda / \alpha} Q_{\beta}(n) A^{\lambda}\left(\eta_{2}(n)\right) y^{\lambda / \alpha}\left(\eta_{2}(n)\right) \leq 0 .
$$

That is, $u(n)=-y(n)$ is a positive solution of an advanced difference equality

$$
\Delta u(n)-N_{1}\left(\frac{\lambda}{(m-2) !}\right)^{\beta}\left(\frac{1}{1+p_{0}^{\beta}}\right)^{\lambda / \alpha} Q_{\beta}(n) A^{\lambda}\left(\eta_{2}(n)\right) u^{\lambda / \alpha}\left(\eta_{2}(n)\right) \geq 0 .
$$

Then, by virtue of [Gyori and Ladas, 1991] the associated advanced difference equation

$$
\Delta u(n)-N_{1}\left(\frac{\lambda}{(m-2) !}\right)^{\beta}\left(\frac{1}{1+p_{0}^{\beta}}\right)^{\lambda / \alpha} Q_{\beta}(n) A^{\lambda}\left(\eta_{2}(n)\right) u^{\lambda / \alpha}\left(\eta_{2}(n)\right)=0 .
$$

also has a positive solution. However, by [Sundar and Murugesan, 2010, Lemma 2.3.2] implies that; under assumption (18), equation (63) is oscillatory. Therefore, equation (1) cannot have positive solutions. This contradiction with our initial assumption complete the proof.

Theorem 9. Let $m \geq 2$ be even and $0<\alpha=\beta \leq 1$. Assume that conditions $\left(c_{4}\right)$ and $\left(c_{5}\right)$ are satisfied, and there exist two real sequences $\eta_{1}(n), \eta_{2}(n)$ satisfying (56). Suppose also that

$$
\frac{1}{((m-1) !)^{\beta}\left(1+p_{0}^{\beta}\right)} \lim \inf _{n \rightarrow \infty} \sum_{\tau^{-1}\left(\eta_{1}(n)\right)}^{n-1} \tilde{Q}_{\beta}(s)>\left(\frac{k}{k+1}\right)^{k+1}
$$


where $k$ denotes the delay arguments and

$$
\frac{1}{((m-2) !)^{\beta}\left(1+p_{0}^{\beta}\right)} \lim _{n \rightarrow \infty} \sum_{n}^{\eta_{1}(n)-1} Q_{\beta}(s) A^{\beta}\left(\eta_{2}(s)\right)>\left(\frac{l-1}{l}\right)^{l}
$$

where $l$ denotes the advance arguments, are satisfied. Then conclusion of Theorem 6 remains infact.

Proof. Assume that $x(n)$ is an eventually positive solution of equation (1) that satisfies (20) and proceeding as in the proof of Theorem 8 , one concludes that; for every $\lambda \in(0,1)$ a delay difference equation

$$
\Delta y(n)+\left(\frac{\lambda}{((m-1) !}\right)^{\beta} \frac{1}{1+p_{0}^{\beta}} \tilde{Q}_{\beta}(n) y\left(\tau^{-1}\left(\eta_{1}(n)\right)\right)=0
$$

and an advanced difference equation

$$
\Delta u(n)-\left(\frac{\lambda}{(m-1) !}\right)^{\beta} \frac{1}{1+p_{0}^{\beta}} Q_{\beta}(n) A^{\beta}\left(\eta_{2}(n)\right) u\left(\eta_{1}(n)\right)=0
$$

have positive solutions. On the other hand, application of condition (64) along with [Gyori and Ladas, 1991] imply that equation (66) is oscillatory, a contradiction. Likewise, by virtue of [Sundar and Murugesan, 2010, Lemma 2.3.2] condition (65) yields that equation (67) is oscillatory. This contradiction completes the proof.

Note that Theorems 6 to 9 ensure that every solution $x(n)$ of equation (1) is either oscillatory or tends to zero as $n \rightarrow \infty$ and unfortunately cannot distinguish solutions with different behaviors. In the remaining part of this section, we establish several results which guarantee that all solutions of equation (1) are oscillatory.

Theorem 10. Let $m \geq 4$ be even and $0<\beta \leq 1$. Assume that conditions $\left(c_{4}\right)$ and $\left(c_{5}\right)$ are satisfied, and there exist there numbers $\gamma, \lambda, \theta \in R$ such that $\gamma \leq \beta \leq \lambda, \gamma<\alpha<\lambda, \theta \geq \beta$ and $\theta>\alpha$. Suppose further that there exist three real sequences $\eta_{1}(n), \eta_{2}(n), \eta_{3}(n)$

$$
\eta_{3}(n) \geq \sigma(n), \quad \eta_{3}(n)>c(n)
$$

and such that (16) holds. Assume that conditions (17), (18) and

$$
\sum^{\infty} Q_{\theta}(n)=\infty
$$

hold. Then equation (1) is oscillatory.

Proof. Without loss of generality, suppose that $x(n)$ is a nonoscillatory solution of equation (1) which is eventually positive. As in the proof of Theorem 6, we obtain (24). In view of equation (1) and Lemma 2, in addition to the case (25), there are two more possible types of behavior of solutions for $n \geq n_{1}$ where $n_{1} \geq n_{0}$ is large enough in the proof of Theorem 6. Namely, one can also have

$$
z(n)>0, \Delta z(n)>0, \Delta^{m-2} z(n)>0, \Delta^{m-1} z(n)<0, \Delta\left[r(n)\left(\Delta^{m-1} z(n)\right)^{\alpha}\right] \leq 0
$$

or

$$
z(n)>0, \Delta^{j} z(n)<0, \Delta^{j+1} z(n)>0, \Delta^{m-1} z(n)<0, \Delta\left[r(n)\left(\Delta^{m-1} z(n)\right)^{\alpha}\right] \leq 0
$$

for all odd integers $j \in\{1,2, \cdots, m-3\}$. However conditions (17) and (18) yields that neither (24) nor (70) is possible. Therefore, we have to analyze the only remaining case, and we assume now that all the conditions in (71) are satisfied. Then, inequality (46) holds. Summing (46) from $n$ to $\infty(n-2)$ times, we obtain

$$
\begin{aligned}
z(n) & \geq-\sum_{n}^{\infty} \frac{(\eta-n)^{(m-3)}}{(m-3) !} A(\eta) r^{1 / \alpha}(n) \Delta^{m-1} z(n) \\
& =-\sum_{n}^{\infty} \frac{(\eta-n)^{(m-3)}}{(m-3) !} A(\eta) w^{1 / \alpha}(n)
\end{aligned}
$$

where $w(n)$ is defined by (30). Taking into account that $\Delta z(n)<0, \sigma(n) \leq \eta_{3}(n)$ and using (24), we have

$$
\Delta\left[r(n)\left(\Delta^{m-1} z(n)\right)^{\alpha}+p_{0}^{\beta} r(\tau(n))\left(\Delta^{m-1} z(\tau(n))\right)^{\alpha}\right]+Q(n) z^{\beta}\left(\eta_{3}(n)\right) \leq 0 .
$$


By virtue of monotonicity of $z(n)$, there exists a constant $M_{2}>0$ such that

$$
z^{\beta}\left(\eta_{3}(n)\right)=z^{\beta-\theta}\left(\eta_{3}(n)\right) z^{\theta}\left(\eta_{3}(n)\right) \geq M_{2} z^{\theta}\left(\eta_{3}(n)\right)
$$

combining (73) and (74), we obtain

$$
\Delta\left[r(n)\left(\Delta^{m-1} z(n)\right)^{\alpha}+p_{0}^{\beta} r(\tau(n))\left(\Delta^{m-1} z(\tau(n))\right)^{\alpha}\right]+M_{2} Q(n) z^{\theta}\left(\eta_{3}(n)\right) \leq 0 .
$$

Using (72) in (75), we conclude that in this case, the sequence $w(n)$ defined by (30) is negative, nonincreasing, and satisfies the inequality

$$
\Delta\left[r(n)\left(\Delta^{m-1} z(n)\right)^{\alpha}+p_{0}^{\beta} r(\tau(n))\left(\Delta^{m-1} z(\tau(n))\right)^{\alpha}\right]+\frac{M_{2}}{((m-3) !)^{\theta}} Q_{\theta}(n) w^{\theta / \alpha}\left(\eta_{3}(n)\right) \leq 0 .
$$

Introduction again $y(n)$ by (33) and using the monotonicity of $w(n)$, we arrive at (48). Substitution of (48) into (76) leads to the conclusion that $y(n)$ is a negative solution of an advanced difference equation

$$
\Delta y(n)-\frac{M_{2}}{((m-3) !)^{\theta}}\left(\frac{1}{1+p_{0}^{\beta}}\right)^{\theta / \alpha} Q_{\theta}(n) y^{\theta / \alpha}\left(\eta_{3}(n)\right) \leq 0 .
$$

In which case the function $u(n)=-y(n)$ is a positive solution of an advanced difference inequality

$$
\Delta u(n)-\frac{M_{2}}{((m-3) !)^{\theta}}\left(\frac{1}{1+p_{0}^{\beta}}\right)^{\theta / \alpha} Q_{\theta}(n) u^{\theta / \alpha}\left(\tau^{-1}\left(\eta_{3}(n)\right)\right) \geq 0 .
$$

Then, by [Gyori and Ladas, 1991], the associated advanced difference equation

$$
u(n)-\frac{M_{2}}{((m-3) !)^{\theta}}\left(\frac{1}{1+p_{0}^{\beta}}\right)^{\theta / \alpha} Q_{\theta}(n) u^{\theta / \alpha}\left(\tau^{-1}\left(\eta_{3}(n)\right)\right)=0,
$$

also has a positive solution. However, by [Sundar and Murugesan, 2010, Lemma 2.3.2] implies that (79) is oscillatory under assumption (69). Therefore, equation (1) cannot have positive solutions. This contradiction with our initial assumption completes the proof.

Theorem 11. Let $m \geq 4$ be even and $0<\alpha=\beta \leq 1$. Assume that conditions $\left(c_{4}\right)$ and $\left(c_{5}\right)$ are satisfied, and that there exist three real sequences $\eta_{1}(n), \eta_{2}(n)$ and $\eta_{3}(n)$ as in Theorem 10. Suppose also that conditions (52) and (55) hold. If

$$
\frac{1}{((m-3) !)^{\beta}\left(1+p_{0}^{\beta}\right)} \lim _{n \rightarrow \infty} \sum_{n}^{\tau^{-1}\left(\eta_{3}(n)\right)-1} \tilde{Q}_{\beta}(s)>\left(\frac{l-1}{l}\right)^{l}
$$

where $l$ denotes the advanced argument, then equation (1) is oscillatory.

Proof. Let $x(n)$ be a nonoscillatory solution of (1) which is eventually positive. As in the proof of Theorem 10, one can have either (25) or (70) or (71). However, conditions (51) and (53) exclude cases (25) and (70). Then all the inequalities in (71) should be satisfied. Along the same lines as in the proof of Theorem 10, one comes to the conclusion that an advanced difference equation

$$
\Delta u(n)-\frac{1}{((m-3) !)^{\beta}\left(1+p_{0}^{\beta}\right)} \bar{Q}_{\beta}(n) u\left(\tau^{-1}\left(\eta_{3}(n)\right)\right)=0
$$

has positive solutions. On the other hand, if condition (80) holds, then by virtue of [Sundar and Murugesan, 2010] implies that equation (80) is oscillatory. This contradiction completes the proof.

Theorem 12. Let $m \geq 4$ be even, $0<\beta \leq 1$, and assume that conditions $\left(c_{4}\right)$ and $\left(c_{5}\right)$ are satisfied. Suppose further that there exist three numbers $\lambda, \gamma, \theta \in R$ as in Theorem 11 and three real sequences $\eta_{1}(n), \eta_{2}(n), \eta_{3}(n)$ such that (56) is satisfied, $\eta_{3}(n) \geq \sigma(n)$ and $\eta_{3}(n) \geq n$. If (17), (18) and (69) hold, then equation (1) is oscillatory.

Proof. Let $x(n)$ be an eventually positive nonoscillatory solution of equation (1). The same argument as in the proof of Theorem 10 yields that (71) holds. Define the sequence $w(n)$ by (30). From the proof of Theorem 10, we already know that $w(n)$ is negative, nonincreasing and satisfies the inequality (76). Introducing the sequence $y(n)$ by (32) and using 
the monotonicity of $w(n)$, we arrive at (60). Substituting (60) into (76) we observe that $y(n)$ is a negative solution of an advanced difference inequality

$$
\Delta y(n)-\frac{M_{2}}{((m-3) !)^{\theta}}\left(\frac{1}{1+p_{0}^{\beta}}\right)^{\theta / \alpha} Q_{\theta}(n) y^{\theta / \alpha}\left(\eta_{3}(n)\right) \leq 0,
$$

while $u(n)=-y(n)$ is a positive solution of an advanced difference inequality

$$
\Delta u(n)-\frac{M_{2}}{((m-3) !)^{\theta}}\left(\frac{1}{1+p_{0}^{\beta}}\right)^{\theta / \alpha} Q_{\theta}(n) u^{\theta / \alpha}\left(\eta_{3}(n)\right) \geq 0 .
$$

In this case, the result due to Gyori [1991] allows one to deduce that the associated advanced difference equation

$$
\Delta u(n)-\frac{M_{2}}{((m-3) !)^{\theta}}\left(\frac{1}{1+p_{0}^{\beta}}\right)^{\theta / \alpha} Q_{\theta}(n) u^{\theta / \alpha}\left(\eta_{3}(n)\right)=0,
$$

also has a positive solution. However, it has been established [Sundar and Murugesan, 2010, Lemma 2.3.2] that if condition (69) is satisfied, then equation (83) is oscillatory. Therefore equation (1) cannot have positive solutions, and this contradiction with the assumptions of the theorem completes the proof.

Theorem 13. Let $m \geq 4$ be even and $0<\alpha=\beta \leq 1$. Assume that conditions $\left(c_{4}\right)$ and $\left(c_{5}\right)$ are satisfied, and there exist three real sequences $\eta_{1}(n), \eta_{2}(n)$ and $\eta_{3}(n)$ as in Theorem 12. Suppose further that (64), (65) hold, and

$$
\frac{1}{((m-3) !)^{\theta}\left(1+p_{0}^{\beta}\right)} \lim _{n \rightarrow \infty} \sum_{n}^{\eta_{3}(n)-1} \bar{Q}_{\beta}(s)>\left(\frac{l-1}{l}\right)^{l}
$$

where $l$ denotes the advanced argument. Then equation (1) is oscillatory.

Proof. Assume that $x(n)$ is an eventually positive nonoscillatory solution of equation (1) and increasing as in the proof of Theorem 10 one concludes that (71) holds. As in the proof of Theorem 12 we observed that an advance difference equation

$$
\Delta u(n)-\frac{1}{((m-3) !)^{\theta}\left(1+p_{0}^{\beta}\right)} \bar{Q}_{\beta}(n) u\left(\eta_{3}(n)\right)=0,
$$

has positive solution. On the other hand, if condition (84) is satisfied, a result reported by [Sundar and Murugesan, 2010, Lemma 2.3.2] yields that equation (85) is oscillatory. This contradiction completes the proof.

\section{Asymptotic Behavior of Solutions to Odd-Order Equations}

In this section, in addition to conditions $\left(c_{4}\right),\left(c_{5}\right)$ and $(10)$, we also assume that

$$
\text { (c) } \sigma(n)<n
$$

The validity of the following four propositions can be established in the same manner as it has been done for Theorems 6 , 9. Therefore, to avoid unnecessary repetition, we only formulate counterparts of Theorems 9 and following for the case of odd-order equations.

Theorem 14. Let $m \geq 3$ be odd and $0<\beta \leq 1$. Assume that conditions $\left(c_{4}\right)-\left(c_{6}\right)$ are satisfied, and there exist two real numbers $\gamma, \lambda \in R$ as in Theorem 6 and a real sequence $\eta_{4}(n)$ such that $n \leq \tau(n)<\eta_{4}(n)$. Suppose further that

$$
\sum^{\infty} \tilde{Q}_{\gamma}(n)=\infty
$$

and

$$
\sum^{\infty} Q_{\beta}(n) A^{\lambda}\left(\eta_{4}(n)\right)=\infty .
$$

Then the conclusion of Theorem 6 remains intact. 
Theorem 15. Let $m \geq 3$ be odd, and let $0<\alpha=\beta \leq 1$. Assume that conditions $\left(c_{4}\right)-\left(c_{6}\right)$ are satisfied, and there exists a real sequence $\eta_{4}(n)$ as in Theorem 14. Suppose also that

$$
\frac{1}{((m-1) !)^{\beta}} \frac{1}{1+p_{0}^{\beta}} \lim _{n \rightarrow \infty} \sum_{\sigma(n)}^{n-1} \widehat{Q}_{\beta}(s)>\frac{1}{e}
$$

and

$$
\frac{1}{((m-2) !)^{\beta}} \frac{1}{1+p_{0}^{\beta}} \lim _{n \rightarrow \infty} \sum_{n}^{\tau^{-1}\left(\eta_{4}(n)\right)-1} Q_{\beta}(s) A^{\beta}\left(\eta_{4}(s)\right)>\frac{1}{e}
$$

Then the conclusion of Theorem 6 remains intact.

Theorem 16. Let $m \geq 3$ be odd and let $0<\beta \leq 1$. Assume that conditions $\left(c_{4}\right)-\left(c_{6}\right)$ are satisfied, and there exist two real numbers $\gamma, \lambda \in R$ as in Theorem 6 and a sequence $\eta_{4}(n)$ such that $\sigma(n)<\tau(n) \leq n \leq \eta_{4}(n)$. Suppose further that conditions (86) and (87) are satisfied. Then the conclusion of Theorem 6 remains intact.

Theorem 17. Let $m \geq 3$ be odd, and $0<\alpha=\beta \leq 1$. Assume that conditions $\left(c_{4}\right)-\left(c_{6}\right)$ are satisfied, and there exists a real sequence $\eta_{4}(n)$ as in Theorem 16. If

$$
\frac{1}{((m-1) !)^{\beta}} \frac{1}{1+p_{0}^{\beta}} \lim _{n \rightarrow \infty} \sum_{\tau^{-1}(\sigma(n))}^{n-1} \widehat{Q}_{\beta}(s)>\frac{1}{e}
$$

and

$$
\frac{1}{((m-2) !)^{\beta}} \frac{1}{1+p_{0}^{\beta}} \lim \inf _{n \rightarrow \infty} \sum_{n}^{\eta_{4}(n)-1} Q_{\beta}(s) A^{\beta} \eta_{4}(s)>\frac{1}{e} .
$$

Then the conclusions of Theorem 6 remain intact.

Note that Theorems 14-17 apply only if $\sigma$ is a delayed argument, $\sigma(n)<n$. Hence it is important to complement such results with the following theorems that can be applied in the case where $\sigma$ is an advanced argument, $\sigma(n) \geq n$.

Theorem 18. Let $m \geq 3$ be odd and let $0<\beta \leq 1$. Assume that conditions $\left(c_{4}\right)$ and $\left(c_{5}\right)$ are satisfied, and there exist two real numbers $\gamma, \lambda \in R$ as in Theorem 6 and two real sequences $\eta_{1}(n), \eta_{2}(n)$ satisfying (16). Suppose also that

$$
\sum_{n_{0}}^{\infty} \xi^{(m-2)}\left[\frac{1}{R(\xi)} \sum_{\xi}^{\infty} Q(s)\right]^{1 / \alpha}=\infty
$$

If (17) and (18) are satisfied, then the conclusions of Theorem 6 remains intact.

Proof. Assume that equation (1) has an eventually positive solution $x(n)$ satisfying (20). Proceeding as in the proof of Theorem 6, we arrive at (23) and observe that equation (1) yields that either (24) or (25) holds. Indeed, it follows from the condition $\Delta\left[r(n)\left(\Delta^{m-1} z(n)\right)^{\alpha}\right] \leq 0$ that either $\Delta^{m-1} z(n)>0$ or $\Delta^{m-1} z(n)<0$. Assume first that $\Delta^{m-1} z(n)<0$; this immediately leads us to conditions (25).

On the other hand, if $\Delta^{m-1} z(n)>0$, then $\Delta^{m} z(n) \leq 0$ to the fact that $\Delta r(n)>0$. We claim that $\Delta z(n)>0$ eventually. Infact, if this is not the case, then $\Delta z(n)<0$ eventually. Since $z(n)>0, \Delta z(n)<0$, and (20) holds, there should exist a positive constant a such that

$$
\lim _{n \rightarrow \infty} z(n)=a
$$

On the other hand, if $\Delta^{m-1} z(n)>0$ and $\Delta^{m} z(n) \leq 0$, there exists a constant $b \geq 0$ such that

$$
\lim _{n \rightarrow \infty} \Delta^{m-1} z(n)=b \geq 0 .
$$

Hence,

$$
\lim _{n \rightarrow \infty} \Delta^{(i)} z(n)=0
$$


for $i=1,2, \cdots, m-1$. Summing (23) from $n$ to $\infty$ and using the fact that the limit

$$
\lim _{n \rightarrow \infty} r(n)\left(\Delta^{m-1} z(n)\right)^{\alpha} \geq 0
$$

is finite, we have

$$
-r(n)\left(\Delta^{m-1} z(n)\right)^{\alpha}-p_{0}^{\beta} r(\tau(n))\left(\Delta^{m-1} z(\tau(n))\right)^{\alpha}+\sum_{n}^{\infty} Q(s) z^{\beta}(\sigma(s)) \leq 0 .
$$

Consequently,

$$
-R(n)\left[\left(\Delta^{m-1} z(n)\right)^{\alpha}+\left(\left(p_{0}^{\beta}\right)^{1 / \alpha}\right)^{\alpha}\left(\Delta^{m-1} z(\tau(n))\right)^{\alpha}\right]+\sum_{n}^{\infty} Q(s) z^{\beta}(\sigma(s)) \leq 0 .
$$

Assume first that $\alpha \leq 1$. Using Lemma 5, we obtain

$$
\begin{aligned}
& \left(\Delta^{m-1} z(n)\right)^{\alpha}+\left(\left(p_{0}^{\beta}\right)^{1 / \alpha}\right)^{\alpha}\left(\Delta^{m-1} z(\tau(n))\right)^{\alpha} \\
& \quad \leq \frac{1}{2^{\alpha-1}}\left[\Delta^{m-1} z(n)+\left(p_{0}^{\beta}\right)^{1 / \alpha} \Delta^{m-1} z(\tau(n))\right]^{\alpha} .
\end{aligned}
$$

Substituting (99) into (98), we have

$$
-2^{1-\alpha} R(n)\left[\Delta^{m-1} z(n)+\left(p_{0}^{\beta}\right)^{1 / \alpha} \Delta^{m-1} z(\tau(n))\right]^{\alpha}+\sum_{n}^{\infty} Q(s) z^{\beta}(\sigma(s)) \leq 0
$$

which implies

$$
-\left[\Delta^{m-1} z(n)+\left(p_{0}^{\beta}\right)^{1 / \alpha} \Delta^{m-1} z(\tau(n))\right]^{\alpha} \leq-\frac{1}{2^{1-\alpha} R(n)} \sum_{n}^{\infty} Q(s) z^{\beta}(\sigma(s)) .
$$

Therefore,

$$
-\left[\Delta^{m-1} z(n)+\left(p_{0}^{\beta}\right)^{1 / \alpha} \Delta^{m-1} z(\tau(n))\right]+\left[\frac{1}{2^{1-\alpha} R(n)} \sum_{n}^{\infty} Q(s) z^{\beta}(\sigma(s))\right]^{1 / \alpha} \leq 0 .
$$

Summing (102) $(m-2)$ times from $n$ to $\infty$ and then one more time from $n_{1}$ to $\infty$. Using (95) and changing the order of summation, we obtain

$$
\sum_{n_{1}}^{\infty} \frac{\left(\xi-\eta_{1}\right)^{(m-2)}}{(m-2) !}\left[\frac{1}{2^{1-\alpha} R(\xi)} \sum_{\xi}^{\infty} Q(s) z^{\beta}(\sigma(s))\right]^{1 / \alpha}<\infty
$$

Inequality (103) yields

$$
\sum_{n_{1}}^{\infty} \xi^{(m-2)}\left[\frac{1}{R(\xi)} \sum_{\xi}^{\infty} Q(s)\right]^{1 / \alpha}<\infty
$$

which contradicts (92).

For the case $\alpha>1$, one arises at the contradiction with the assumptions of the theorem by using Lemma 4 . Then we conclude that $\Delta z(\alpha)>0$ eventually. The rest of the proof follows the same lines as in Theorem 6 and is omitted.

Combining the ideas exploited in the proofs of Theorems 7-9 and 18, one can derive the following results.

Theorem 19. Let $m \geq 3$ be odd, and let $0<\alpha=\beta \leq 1$. Assume that conditions $\left(c_{4}\right)$ and $\left(c_{5}\right)$ are satisfied, and there exist two real sequence $\eta_{1}(n), \eta_{2}(n)$ satisfying (16). If (52), (53) and (92) hold, then the conclusion of Theorem 6 remains intact.

Theorem 20. Let $m \geq 3$ be odd, and $0<\beta \leq 1$. Assume that conditions $\left(c_{4}\right)$ and $\left(c_{5}\right)$ are satisfied, and there exist two real numbers $\gamma, \lambda \in R$ as in Theorem 6 and two real sequences $\eta_{1}(n), \eta_{2}(n)$ satisfying (56). If conditions (17) and (18) and (92) are satisfied, the conclusion of Theorem 6 remains intact. 
Theorem 21. Let $m \geq 3$ be odd, and $0<\alpha=\beta \leq 1$. Assume that conditions $\left(c_{4}\right)$ and $\left(c_{5}\right)$ are satisfied, and there exist two real sequences $\eta_{1}(n), \eta_{2}(n)$ satisfying (56). If conditions (64) and (65) and (92) are satisfied, then the conclusion of Theorem 6 remains intact.

\section{Examples and Discussions}

The following examples illustrate applications of some of theoretical results presented in the previous sections. In all the examples, $p_{0}$ is a constant such that $0 \leq p_{0}<\infty$.

Example 1. For $n \geq 1$, consider the fourth order neutral difference equation

$$
\Delta\left(e^{n}\left(\Delta^{3}\left(x(n)+p_{0} x(n-2)\right)\right)\right)+\left(1+p_{0} e\right)(1-\sqrt{e})^{3}\left(\frac{1}{\sqrt{e}}-1\right) e^{n-\frac{3}{2}} x(n-1)=0
$$

Let $\eta_{1}(n)=n-3$ and $\eta_{2}(n)=n+1$. An application of Theorem 9 yields that every solution $x(n)$ of equation (105) is either oscillatory or satisfies $\lim _{n \rightarrow \infty} x(n)=0$. As a matter of that $x(n)=e^{-\frac{n}{2}}$ is an exact solution to $(105)$ satisfying $\lim _{n \rightarrow \infty} x(n)=0$.

Example 2. For $n \geq 1$, consider a fourth order neutral difference equation

$$
\Delta\left(e^{n}\left(\Delta^{3}\left(x(n)+p_{0} x(n+2)\right)\right)\right)+\left(e^{2}+1\right)\left(1+e^{3}\right)\left(1+p_{0} e\right)^{2} e^{n+3} x(n-3)=0 .
$$

Let $\eta_{1}(n)=n-3$ and $\eta_{2}=\eta_{3}=n+3$. Using Theorem 11, we deduce that equation (106) is oscillatory. It is easy to verify that one oscillatory solution of the equation $x(n)=(-1)^{n} e^{n}$.

Example 3. For $n \geq 1$, consider a third order neutral difference equation

$$
\Delta\left(e^{n}\left(\Delta^{2}\left(x(n)+p_{0} x(n-2)\right)\right)\right)+\left(1+p_{0} e\right)\left(\frac{1}{e}-1\right)^{2}(1-\sqrt{e}) e^{n-\frac{1}{2}} x(n-1)=0 .
$$

Let $\eta(n)=n+1$. It follows from Theorem 17 that every solution $x(n)$ of equation (107) is either oscillatory or satisfies $\lim _{n \rightarrow \infty} x(n)=0$ is $x(n)=e^{-\frac{n}{2}}$.

Remark 1. By using inequality

$$
x_{1}^{\beta}+x_{2}^{\beta} \geq 2^{1-\beta}\left(x_{1}+x_{2}\right)^{\beta}
$$

which holds for any $\beta \geq 1$ and for all $x_{1}, x_{2} \in[0, \infty)$, results reported in this paper can be extended to equation (1) for all $\beta \in R$ which satisfy $\beta>1$. In this case one has to replace $Q(n)=\min \{q(n), q(\tau(n))\}$ with a function $Q(n)=$ $2^{1-\beta} \min \{q(n), q(\tau(n))\}$ and proceed in above.

Remark 2. Our main assumptions on functional arguments do not specify whether $\tau(n)$ is delayed or an advanced argument. Remarkably, $\sigma(n)$ can even switch its nature between an advanced and delayed argument. However, such flexibility is achieved at the cost of requiring that the function $\tau$ is monotonic and satisfies $\tau \circ \sigma=\sigma \circ \tau$. The question regarding the analysis of the asymptotic behavior of solutions to (1) with other methods that do not require these assumptions remains open at the moment.

\section{Acknowledgements}

The authors wish to express their sincere thanks to the referee for valuable comments and suggestions.

\section{References}

Agarwal. R. P. (1992). Difference Equations and Inequalities Theory, Method and Applications. Marcel Dekker, New York.

Agarwal. R. P. \& Wong. P. J. Y. (1997). Advanced Topics in Difference Equations. Kluwer Academic Publishers Grovi, Dordrecht.

Bolat, Y., \& Alzabut, J. O. (2012). On the oscillation of higher-order half-linear delay difference equations. Appl. Math. Int. Sci., 6, 423-427.

Cheng, S. S., \& Patula, W. T. (1993). An existence theorem for a nonlinear difference equations, Nonlinear Analysis, Theory Method and Applications, 20(3), 193-203.

Elaydi, S. N. (1995). An Introduction to Difference Equations. Springer Verlag, New York.

Graef, J. R, Mician, A. S, Spokes, P. W, Sundar, P., \& Thandapani, E. (1996). Oscillatory and asymptotic behavior of neutral type difference equation. J. Austra. Math. Soc. Ser. B., 38, 163-171. https://doi.org/10.1017/S0334270000000552 
Guan, X., \& Yang, J. (1999). Asymptotic and oscillatory behavior of higher-order nonlinear neutal difference equations, Kyun. Math. I., 39, 251-259.

Gyori, I., \& Ladas, G. (1991). Oscillation Theory of Delay Differential Equations with Applications. Clarendon Press, Oxford.

He, X. Z. (1993). Oscillatory and Asymptotic behavior of second order nonlinear difference equations. J. Math. Anal. Appl., 175, 482-498. https://doi.org/10.1006/jmaa.1993.1186

Jurang, Y., \& Bin, L. (1997). Oscillatory and asymptotic behavior of fourth order nonlinear difference equations. Acta. Math. Sinica. New Series, 13, 105-115. https://doi.org/10.1007/BF02560530

Karpuz, B. (2009). On Oscillation and asymptotic behavior of a higher order functional difference equation of neutral type, Inter. J. Differen. Eqn., 4(1), 69-96.

Kubiaczyk, I., \& Sekar, S. H. (2002). Oscillation theorems for second order sublinear delay difference equations. Math. Slovaca, 52, 343-359.

Lakshmikanthan, V., \& Trigiante, O. (1988). Theory of Difference Equations: Numerical Method and Application. Academic Press, New York.

Luo, J. (2002). Oscillation criteria for second order quasilinear neutral difference equations. Computers Math. Appl., 43, 1549-1557. https://doi.org/10.1016/S0898-1221(02)00118-9

Ou, C. H., \& Wong, J. S. W. (2004). Oscillation and nonoscillation theorems for superlinear Emden-Fowler equations of the fourth order. Annali di Mathematica Pura e Applicata, Series IV, 183(1), 25-43. https://doi.org/10.1007/s10231003-0079-Z

Sundar, P., \& Kishorkumar. (2014). Nonoscillatory solution of even-order nonlinear neutral difference equations. Journal of Indian Academy of Mathematics, 36(1).

Sundar, P., \& Kishorkumar. (2014). Oscillation results for even-order quasilinear neutral functional difference equation, IOSR Journal of Mathematics, 10(4), 8-18.

Sundar, P., \& Murugesan, A. (2010). Asymptotic Behavior and Oscillation of Solutions of Neutral/Non-Neutral Advanced Difference Equations, Ph.D., Dissertation, Periyar University, Tamil Nadu, India, October.

Sundar, P., \& Thandapani, E. (2000). Oscillation and non-oscillation theorems for second order quasilinear functional difference equations. Indian J. Pure Appl. Math., 31(1), 37-47.

Wong, J. S. W. (1975). On the generalized Emden-Fowler equation. SIAM Review, 17, 339-360. https://doi.org/10.1137/1017036

Yu, J. S., \& Wang, Z. C. (1994). Asymptotic behavior and oscillation in neutral delay difference equations. Funk. Ekvac., $37,241-248$.

Yildiz, M. K., \& Ocalan, O. (2007). Oscillation results for higher order nonlinear neutral delay difference equation. Appl. Math. Lett., 20(3), 243-247. https://doi.org/10.1016/j.aml.2006.05.001

Zafer, A. (1995). Oscillation and asymptotic behavior of higher order difference equations. Math. Comput. Modelling, 2l(4), 43-50. https://doi.org/10.1016/0895-7177(95)00005-M

Zhou, X., \& Yan, J. (1998). Oscillatory and asymptotic behavior of higher order nonlinear difference equations. Nonlinear Analysis, Theory Methods and Applications, 31(3-4), 493-502. https://doi.org/10.1016/S0362-546X(97)00417-3

\section{Copyrights}

Copyright for this article is retained by the author(s), with first publication rights granted to the journal.

This is an open-access article distributed under the terms and conditions of the Creative Commons Attribution license (http://creativecommons.org/licenses/by/4.0/). 